\title{
ON REE'S SERIES OF SIMPLE GROUPS ${ }^{1}$
}

\author{
BY HAROLD N. WARD ${ }^{2}$
}

Communicated by A. M. Gleason, August 9, 1962

Ree recently discovered a series of finite simple groups related to the simple Lie algebra of type $\left(G_{2}\right)[5 ; 6]$. We have determined the irreducible characters of these groups. In this work, we do not use the actual definition of Ree's groups, but only the properties (1)-(5) given below. Since these are sufficient to determine the bulk of the character tables of these groups, it is our hope that they actually characterize the groups completely.

The five properties used are

(1) If $G$ is one of these groups, the order of $G$ is even and the 2Sylow subgroup of $G$ is elementary Abelian of order 8 .

(2) $G$ has no normal subgroup of index 2 .

(3) There is an involution $J$ (an element of order 2) in $G$ such that if $C(J)$ is the centralizer of $J$ in $G$ and $\langle J\rangle$ is the group generated by $J$, then $C(J) /\langle J\rangle$ is isomorphic to $L F(2, q)$, the linear fractional group in two variables over a field of $q$ elements. Here we restrict $q$ by $q \equiv 3(\bmod 8)$ and $q \geqq 27$.

It follows that $C(J)$ is the direct product of $\langle J\rangle$ and a subgroup $F$ of $G$ which is isomorphic to $L F(2, q)$. In $F$ there is an element $R$ of order $(q-1) / 2$, and we also have

(4) If $R^{a} \neq 1$, then $C\left(R^{a}\right) \subseteq C(J)$.

Finally, there is an element $S$ of $F$ with order $(q+1) / 2$. Let $J^{\prime}=S^{t}$ where $t=(q+1) / 4$. Then $S^{2}$ generates the commutator subgroup of $C\left(J, J^{\prime}\right)$, the centralizer of $\left\langle J, J^{\prime}\right\rangle$. We have

(5) For $S^{2}, C\left(S^{2}\right) \subseteq C(J)$.

This condition can actually be replaced by the weaker condition

$\left(5^{*}\right)$ Let $A \in N\left\langle J, J^{\prime}\right\rangle$, the normalizer in $G$ of $\left\langle J, J^{\prime}\right\rangle$, but $A \notin$ $C\left(J, J^{\prime}\right)$. Let $A^{3}=1$. Then $A$ does not commute with $S^{2}$.

From these conditions on $G$ we derive a number of results. The approach is almost entirely by means of characters, both ordinary and modular. Condition (4) leads to two families of exceptional characters related to the classes of $R^{a}(\neq 1)$ and $J R^{a}(\neq J)$. These characters are the characters of 2-defect 1 . Results of Brauer $[1 ; 2 ; 3]$ then lead to two possibilities for the principal 2-block (the 2-block containing the character which is 1 everywhere); one contains seven

1 This note is a summary of the results obtained in the author's Harvard University dissertation of the same title, written under the direction of Richard Brauer.

2 This research was supported by the National Science Foundation. 
ordinary characters, the other contains eight. By means of formulas in [4], relating the order of $C(J)$ to the order of $G$ through generalized decomposition numbers, the case of seven characters may be ruled out. These formulas are applied to the principal 2-block and to a 2block of defect 2 containing four ordinary characters (whose existence is guaranteed by condition (5)). Another application of these formulas then shows that the order of $G$ is $q^{3}(q-1)\left(q^{3}+1\right)$, where $q$ is the $q$ of condition (3) and must moreover be an odd power of 3 .

The characters of 2-defect 2 are shown to make up two more families of exceptional characters (except when $q=27$, when only one family is involved; one of these characters is no longer exceptional). These characters involve the classes of the $S^{a}(\neq 1)$. At this point enough information exists about the values of the characters of positive 2-defect to show that $G$ has a doubly transitive permutation representation of degree $q^{3}+1$ on the cosets of the normalizer of a 3-Sylow subgroup. Here we also show that the center of the 3-Sylow subgroup is elementary Abelian of order $q$ and that its commutator subgroup and Frattini subgroup coincide and have order $q^{2}$.

Next we show that there are two more families of exceptional characters corresponding to classes of elements with orders dividing $q^{2}-q$ +1 . These characters are of 2 -defect 0 , and they and the characters given so far account for all but two of the irreducible characters of $G$. The last two characters are complex conjugates and have 2-defect 0 . The character table of $G$ can now be completed, except for certain values of the various exceptional characters on their corresponding classes. The table shows that $G$ is simple.

\section{REFERENCES}

1. R. Brauer, Zur Darstellungstheorie der Gruppen endlicher Ordnung, Math. Z. 63 (1956), 406-444.

2. - Zur Darstellungstheorie der Gruppen endlicher Ordnung. II, Math. Z. 72 (1959), 25-46.

3. - On blocks of representations of finite groups, Proc. Nat. Acad. Sci. 47 (1961), 1888-1890.

4. - Investigations on groups of even order. I, Proc. Nat. Acad. Sci. 47 (1961), 1891-1893.

5. R. Ree, $A$ family of simple groups associated with the simple Lie algebra of type $\left(G_{2}\right)$, Bull. Amer. Math. Soc. 66 (1960), 508-510.

6. - - A family of simple groups associated with the simple Lie algebra of type $\left(G_{2}\right)$, Amer. J. Math. 83 (1961), 432-462.

HARVARD UNIVERSITY 